\title{
ETAPAS DEL CAMBIO, MOTIVACIONES Y BARRERAS RELACIONADAS CON EL CONSUMO DE FRUTAS Y VERDURAS Y LA ACTIVIDAD FÍSICA EN MADRES DE PREESCOLARES ATENDIDAS EN CENTROS DE ATENCIÓN PRIMARIA DE SALUD
}

\author{
STAGES OF CHANGE, MOTIVATIONS AND BARRIERS \\ RELATED TO THE CONSUMPTION OF FRUIT AND VEGETABLES \\ AND PHYSICAL ACTIVITY IN MOTHERS OF PRESCHOOL \\ CHILDREN ATTENDING PRIMARY HEALTH CENTERS
}

\author{
Lorena Meléndez I. (1), Sonia Olivares C. (2), \\ Lydia Lera M. (2), Fernanda Mediano S. (3)
}

(1) Programa de Magíster, INTA, Universidad de Chile. Santiago, Chile.

(2) Instituto de Nutrición y Tecnología de los Alimentos (INTA), Universidad de Chile. Santiago, Chile.

(3) Centro de Salud Familiar Salvador Bustos, La Florida, Santiago. Chile.

\begin{abstract}
In order to design effective interventions for the prevention of childhood obesity the stages of change, motivations and barriers related to the consumption of five daily portions of fruit and vegetables and physical activity were determined in 323 mothers of preschool children attending Primary Health Centers, through the application of a previously validated survey. Only 5\% of them consumed 5 or more portions with the majority (61.3\%) consuming 1 to 2 portions. The main motivations to eat 5 portions were "I like them" and "to lose weight" while the main barrier was "I forget to eat them". With respect to physical activity, $33.1 \%$ considered themselves on the maintenance stage and $18.9 \%$ on the action stage. Main motivations were: "Makes me feel good", "To lose weight". Main barrier: "I don't have time". It is suggested that educational interventions consider the mother attitudes and beliefs to be more effective.
\end{abstract}

Key words: Preschool mothers, motivations, barriers, fruit and vegetables consumption, physical activity.

Este trabajo fue recibido el 24 de Mayo de 2011 y aceptado para ser publicado el 1 de octubre de 2011.

\section{INTRODUCCIÓN}

En las últimas décadas, los profundos cambios experimentados en la alimentación y los estilos de vida de la población, se han traducido en un significativo aumento en la prevalencia de obesidad y enfermedades no transmisibles asociadas a ella, como la hipertensión, la diabetes, las enfermedades cardiovasculares y algunos tipos de cáncer (1). Esto ha llevado a la Organización Mundial de la Salud (OMS) a proponer una estrategia mundial orientada a su prevención y control (2).

En Chile, la segunda Encuesta Nacional de Salud 2009-2010 (3), realizada en una muestra representativa de la población adulta estableció que, en relación a la Encuesta realizada en el año 2003 (4), la proporción de personas con sobrepeso y obesidad aumentó desde el 61 al 67\%. La proporción fue mayor en las mujeres que en los hombres y más alta en las con menor nivel educacional. La mayor prevalencia en el grupo de menor nivel educacional se observó también en hipertensión, diabetes y riesgo cardiovascular. El año 2009-2010, el sedentarismo afectaba al 92,9\% de las mujeres, cifra que en la Encuesta de Calidad de Vida y Salud del año 2006 alcanzó al 91\% (5).

Por otra parte, la prevalencia de obesidad infantil alcanzó al 23,1\% en los niños que ingresaron a primer año básico en las escuelas públicas del país el año 2010, constituyendo en la actualidad el principal problema de salud pública en este grupo de población (6). En los 
menores de 6 años atendidos en los Servicios de Salud, la prevalencia de sobrepeso alcanzó al 22,4\% y la de obesidad al 9,4\% el año 2009 (7). Un estudio que desagregó la prevalencia de obesidad en preescolares asistentes a la Junta Nacional de Jardines Infantiles (JUNJI) $(8,9)$, mostró un aumento significativo entre los 2 y 3 años $(12,5$ a $18,9 \%)$ y entre los 3 y 4 años $(18,9$ a $20,1 \%)$.

La evidencia sobre la relación entre el peso, la alimentación de tipo occidental y la falta de actividad física con las enfermedades no transmisibles, incluye también diversos tipos de cáncer. Se estima que los factores alimentarios explican aproximadamente el 30\% de los cánceres en países industrializados y, en forma específica, se ha señalado que el consumo de al menos $400 \mathrm{~g}$ diarios de frutas y verduras disminuye el riesgo de varios tipos de cáncer y de enfermedades cardiovasculares $(1,10)$.

Esto justifica las acciones que incentivan el aumento del consumo de frutas y verduras en la población, realizadas por la Corporación 5 al Día Chile $(11,12)$ y las actividades realizadas por el Ministerio de Salud para difundir las Guías para una Vida Saludable o prevenir la obesidad en los niños $(13,14)$. Estas acciones de promoción, si bien han sido un aporte a la información de la población, no han considerado la implementación de estrategias que consideren las etapas del cambio de conducta en las que se encuentran los distintos grupos, así como las motivaciones y barreras que afectan su comportamiento, aspectos necesarios para mejorar la efectividad de las intervenciones (15-18).

Los modos de alimentarse, las preferencias y rechazos hacia determinados alimentos están fuertemente relacionados con el contexto familiar durante la etapa infantil, en la que se incorpora la mayoría de los hábitos alimentarios. En la infancia, la madre es la principal responsable de la transmisión al hijo de pautas alimentarias saludables que podrían prevenir enfermedades relacionadas con la alimentación (19-21).

El objetivo de este trabajo fue determinar las etapas del cambio de conducta y la percepción de las motivaciones y barreras respecto al consumo de frutas y verduras y la realización de actividad física en madres de preescolares de nivel socioeconómico (NSE) medio bajo, de distinto estado nutricional, asistentes a Centros de Atención Primaria de Salud. Se espera contribuir con información de base para la implementación de futuras estrategias educativas más efectivas para la prevención y control de la obesidad desde etapas tempranas de la vida.

\section{SUJETOS Y MÉTODO}

Se realizó un estudio descriptivo de corte transversal, en el que se aplicó una encuesta sobre etapas del cambio de conducta, motivaciones y barreras respecto al consumo de 5 porciones diarias de frutas y verduras $(\mathrm{F} \& \mathrm{~V})$ y la realización de actividad física (AF) a 323 madres de preescolares de NSE medio-bajo en las ciudades de Concepción y Santiago. La muestra se determinó con un nivel de confianza del $95 \%$ y una precisión del $5 \%$, considerando una prevalencia de obesidad de $30 \%$, correspondiente a las mujeres de este NSE.

Las madres de los preescolares fueron seleccionadas entre las que asistían a control de niño sano en los Centros de Salud Familiar (CESFAM) Nonguén de Concepción y San Luis de Peñalolén, en Santiago, ambos ubicados en sectores de bajos ingresos. Las madres fueron invitadas a participar con la autorización de la dirección de cada establecimiento de atención primaria. Las madres que aceptaron, firmaron el consentimiento informado aprobado por el Comité de Ética del INTA, de la Universidad de Chile.

El NSE de la muestra se determinó aplicando la encuesta ESOMAR, actualmente utilizada en estudios poblacionales en el país (22). Todas las madres pertenecían al NSE medio-bajo.

La encuesta fue respondida en forma voluntaria por las madres, a quienes se solicitó que colocaran sus antecedentes personales (fecha de nacimiento, escolaridad, ocupación). Una nutricionista entrenada midió la estatura y peso de las madres.

El modelo transteórico o de etapas del cambio de conducta, propuesto por Prochaska y cols. (17), clasifica a las personas en las etapas de: Precontemplación, cuando la persona considera que no tiene necesidad de cambiar; Contemplación, cuando la persona reconoce la existencia de un problema y manifiesta su intención de cambio en los próximos seis meses; Preparación para la acción, cuando la persona tiene la seria intención de actuar dentro del próximo mes; Acción, cuando el cambio de conducta se ha iniciado y tiene menos de 6 meses y Mantenimiento, cuando el cambio de conducta se ha mantenido durante seis meses o más.

Para evaluar el consumo de frutas y verduras y las etapas del cambio de conducta referidas al tema, se utilizó el cuestionario diseñado y validado por el Programa 5 al Día del Instituto Americano de Investigación del Cáncer (AICR) (23), actualmente utilizado en el Programa 5 al Día Chile, previa adaptación y validación realizada por Zacarías y cols. (24).

Para evaluar las etapas del cambio sobre actividad física, se utilizó la pregunta elaborada por Marcus B y cols. (25), a partir de los criterios de Prochaska (17), actualmente utilizada por el Ministerio de Salud (26).

Para la elaboración de las preguntas y opciones de respuesta del cuestionario con las que se determinaron las percepciones sobre las motivaciones y barreras en 
relación al consumo de 5 porciones diarias de $\mathrm{F} \& \mathrm{~V}$, y la realización de al menos 30 minutos de actividad física 5 veces por semana, se utilizó el cuestionario elaborado por Olivares y cols. $(27,28)$, a partir del análisis de contenido de grupos focales con los que se validó el mensaje sobre F\&V de la Guía para una Vida Saludable 2005 (14) y estudios internacionales $(29,30)$.

Se supervisó la aplicación del instrumento y al final de cada jornada se revisó la codificación de la información en el $100 \%$ de los cuestionarios.

Con los datos de peso y estatura tomados por la nutricionista, se calculó el índice de masa corporal (IMC) y clasificó el estado nutricional utilizando los criterios recomendados por la OMS para adultos: bajo peso, IMC $<18,5$; peso normal, IMC $\geq 18,5$ a 24,9; sobrepeso, IMC $\geq 25$ a 29,9 y obesidad, IMC $\geq 30$ (31).

El peso y estatura de los hijos preescolares de las madres del estudio fueron obtenidos del último control registrado en el CESFAM, realizado por enfermeras y nutricionistas de los respectivos centros de salud. Para estimar el estado nutricional de los niños se utilizó la clasificación de la OMS (32), según las normas del Ministerio de Salud.

Con el fin de profundizar la información sobre las percepciones, valores, creencias y mensajes que podrían convencerlas para consumir 5 porciones diarias de frutas y verduras y realizar actividad física, se invitó a las madres a participar en grupos focales de seis integrantes cada uno. Antes de su realización se les explicó el objetivo y se les aseguró la confidencialidad y adecuada utilización de sus opiniones. La guía para los grupos focales incluyó preguntas sobre los temas citados, para lo que se contó con el apoyo técnico de un psicólogo. Se estimó un número de grupos suficiente para alcanzar el nivel de saturación teórica del contenido (cuando los datos empiezan a repetirse y ya no surgen nuevos aportes), lo que se logró al cuarto grupo.

\section{Análisis estadístico}

Se realizó un análisis descriptivo de las variables analizadas. Las variables continuas se expresan como promedios \pm desviación estándar (DE) y las variables categóricas se describen mediante frecuencias relativas expresadas en porcentajes. Para analizar la fiabilidad del cuestionario se realizó una evaluación de la consistencia interna de sus dimensiones mediante el coeficiente alpha de Cronbach. Para evaluar la asociación entre el estado nutricional, el consumo de frutas y verduras, y las motivaciones y barreras, según género o NSE, se utilizaron la prueba de chi-cuadrado o la prueba exacta de Fisher.

Todos los análisis estadísticos se realizaron utilizando el programa STATA, versión 10.1 (33).

\section{RESULTADOS}

En la tabla 1 se presentan las características de la muestra. Debido a que no se encontraron diferencias

\section{TABLA 1}

\section{Características de las madres de preescolares estudiadas}

\begin{tabular}{lc} 
Mujeres & $\mathbf{n}=\mathbf{3 2 3}$ \\
\hline Edad (años) (promedio \pm DE) & $31,6 \pm 4,9$ \\
NSE & Medio bajo \\
IMC (promedio \pm DE) & $26,9 \pm 4,9$ \\
Estado nutricional \% (n) & \\
Bajo peso & $0,9(3)$ \\
Peso normal & $35,9(116)$ \\
Sobrepeso & $40,6(131)$ \\
Obesas & $22,6(73)$ \\
& \\
Niños n (\%) & Características de los hijos de las madres estudiadas \\
Niñas n (\%) & $155(48 \%)$ \\
Edad (años) (promedio \pm DE) & $168(52 \%)$ \\
IMC (promedio \pm DE) & $3,4 \pm 0,9$ \\
DE: Desviación estándar & $16,9 \pm 1,8$ \\
\hline
\end{tabular}


significativas entre las dos comunas, los resultados se presentan agrupados.
Sólo el $0,9 \%$ de las madres presentaba bajo peso, el $35,9 \%$ estado nutricional normal, el 40,6\% sobrepeso y

\section{FIGURA 1}

Estado nutricional de preescolares según estado nutricional de sus madres.

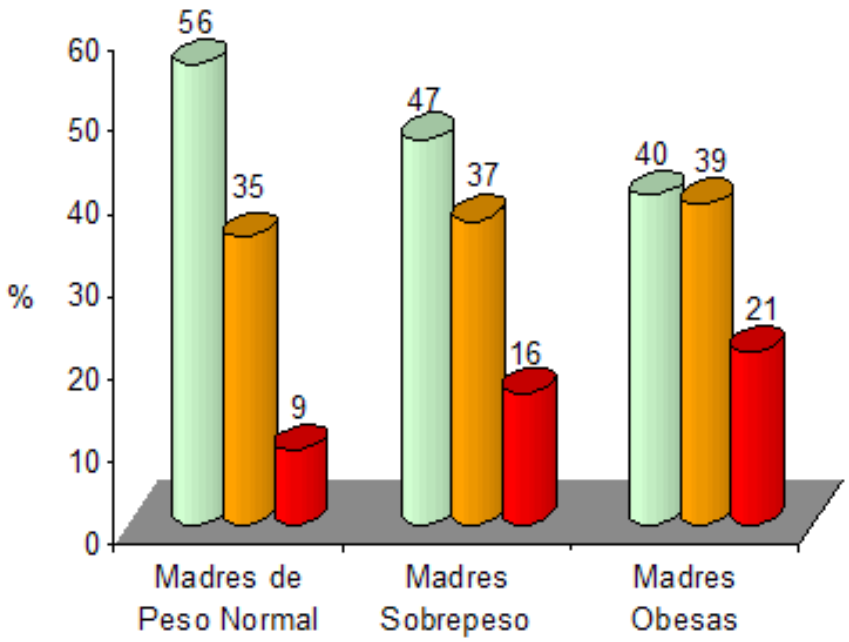

$\square$ Hijos de Peso Normal

$\square$ Hijos Sobrepeso

$\square$ Hijos Obesos

$\mathrm{Chi}^{2}=6.89 \mathrm{p}=0.141$

FIGURA 2

Consumo de frutas y verduras en madres de preescolares.

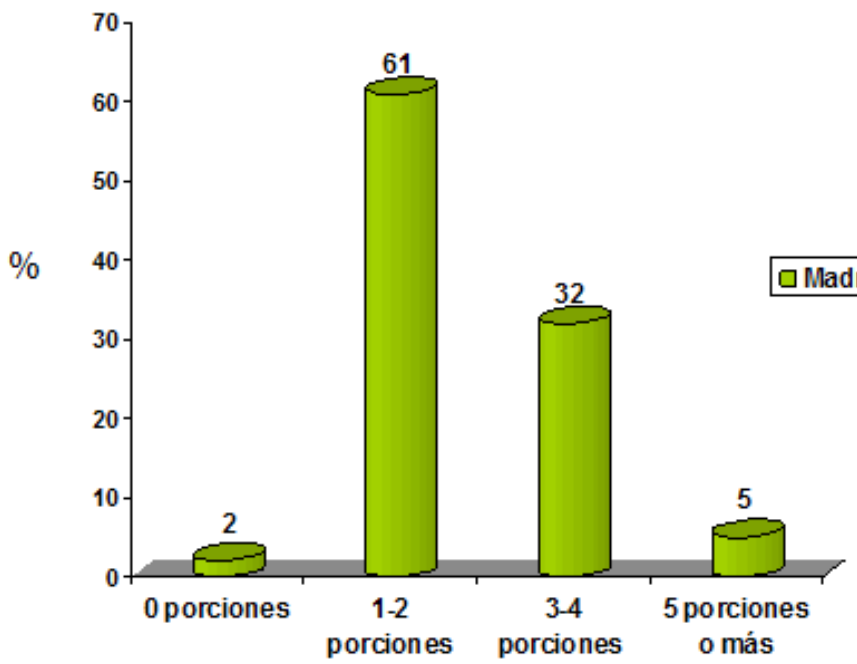


el 22,6\% obesidad. Por su escaso número, las madres con bajo peso no fueron incluidas en al análisis de los resultados.

De los 323 hijos en edad preescolar, la edad promedio fue de 3,4 \pm 0,9 años y el IMC promedio de 16,9 \pm 1,8. Al relacionar el estado nutricional de los preescolares con el estado nutricional de sus madres, en la figura 1 se observa que el $9 \%$ de los hijos de madres de peso normal tenía obesidad, cifras que aumentaron al 14\% en los hijos de madres con sobrepeso y al $21 \%$ en los hijos de madres obesas. El sobrepeso resultó similar en los tres grupos (35 a 39\%). Las diferencias no fueron significativas.

El cuestionario aplicado a las madres presentó una buena consistencia interna (coeficiente de fiabilidad $=$ $0,75)$,

En el total de madres, en la figura 2 se observa que sólo un $5 \%$ consumía las 5 porciones diarias de frutas y verduras recomendadas por la OMS, las que se encontraban en las etapas de acción $(0,9 \%)$ y mantenimiento $(4,1 \%)$. No se encontraron diferencias significativas en el consumo de frutas y verduras según el estado nutricional de las madres.

$\mathrm{Al}$ analizar las etapas del cambio en el grupo mayoritario, que comía $1-2$ porciones $(61,3 \%)$, el $1 \%$ se encontraba en la etapa de pre-contemplación, el 4,9\% en la etapa de contemplación y el $94,1 \%$ en la etapa de preparación para la acción (datos no mostrados).

Las motivaciones y barreras de las madres de preescolares para consumir 5 porciones de frutas y verduras al día fueron analizadas según estado nutricional, para determinar si existían algunas diferencias útiles para el diseño de mensajes y programas educativos. En la tabla 2 se observa que las motivaciones más frecuentes en ambos grupos fueron "me gustan", "para prevenir enfermedades", "me facilitan la digestión" y "me hacen

\section{TABLA 2}

\section{Motivaciones y barreras de madres de preescolares de educación básica para comer 5 porciones diarias de frutas y verduras, según estado nutricional. $(\mathrm{N}=320)$.}

\begin{tabular}{|c|c|c|c|c|c|c|}
\hline \multirow{2}{*}{ MOTIVACIONES } & \multicolumn{2}{|c|}{$\begin{array}{l}\text { Peso normal } \\
\quad n=116\end{array}$} & \multicolumn{2}{|c|}{$\begin{array}{l}\text { Sobrepeso y obesas } \\
\qquad n=204\end{array}$} & \multicolumn{2}{|c|}{$\begin{array}{c}\text { Total } \\
\mathbf{n}=\mathbf{3 2 0}\end{array}$} \\
\hline & $\mathbf{n}$ & $\%$ & $\mathbf{n}$ & $\%$ & $\mathbf{n}$ & $\%$ \\
\hline Me gustan & 87 & 75,0 & 137 & 67,2 & 225 & 70,0 \\
\hline Para prevenir enfermedades & 54 & 46,6 & 99 & 48,5 & 153 & 47,8 \\
\hline Me facilitan la digestión & 61 & 52,6 & 92 & 45,1 & 153 & 47,8 \\
\hline Me hacen sentir bien & 46 & 39,6 & 66 & 32,6 & 112 & 35,0 \\
\hline Para bajar de peso & 28 & 24,1 & 96 & 47,1 & 124 & $38.8^{*}$ \\
\hline Para estar en forma & 25 & 21,6 & 50 & 24,5 & 75 & 23,4 \\
\hline Para mantener mi peso actual & 36 & 31,0 & 35 & 17,2 & 71 & 22,2 \\
\hline \multicolumn{7}{|l|}{ BARRERAS } \\
\hline & $\mathbf{n}$ & $\%$ & $\mathbf{n}$ & $\%$ & $\mathbf{n}$ & $\%$ \\
\hline Se me olvida comerlas & 51 & 44,0 & 60 & 29,4 & 111 & $34,7 *$ \\
\hline Son caras & 31 & 26,7 & 66 & 32,4 & 97 & 30,3 \\
\hline No acostumbro comerlas & 21 & 18,1 & 45 & 22,1 & 66 & 20,6 \\
\hline No me quitan el hambre & 17 & 14,7 & 38 & 18,6 & 55 & 17,2 \\
\hline No tengo tiempo & 16 & 13,8 & 38 & 18,6 & 54 & 16,9 \\
\hline Sólo como en primavera-verano & 15 & 12,9 & 38 & 18,6 & 53 & 16,6 \\
\hline Me da flojera prepararlas & 13 & 11,2 & 27 & 13,2 & 40 & 12,5 \\
\hline Son difíciles de llevar al trabajo & 5 & 4,3 & 14 & 6,9 & 19 & 5,9 \\
\hline Prefiero los alimentos más calóricos & 2 & 1,7 & 15 & 7,4 & 17 & $5,3 *$ \\
\hline No me gustan & 7 & 6,0 & 10 & 4,9 & 17 & 5,3 \\
\hline
\end{tabular}


sentir bien", sin diferencias significativas según estado nutricional. La opción "para bajar de peso" obtuvo una mayor proporción de respuestas en las madres con sobrepeso y obesas $(\mathrm{p}<0,05)$.

En cuanto a las barreras, la respuesta más frecuente "se me olvida comerlas" fue significativamente mayor en las madres de peso normal $(\mathrm{p}<0,05)$, seguida por "son caras", "no acostumbro a comerlas", "no me quitan el hambre" y "no tengo tiempo", sin diferencias significativas.

En los grupos focales para determinar las percepciones, creencias y valores sobre el consumo de 5 porciones de frutas y verduras y su relación con la alimentación saludable, las madres señalaron "recomendaría comer frutas y verduras, pescado y legumbres a una persona que quiere alimentarse en forma saludable". Sin embargo, destacaron que los alimentos que no podían faltar en la casa eran "la leche, el yogurt y otros lácteos", y "por nuestros hábitos no puede faltar el pan". Con respecto a sus dificultades para consumir las 5 porciones recomendadas por la OMS destacaron "muchas veces por tiempo, no se alcanzan a comer 5 porciones", "el problema es que los niños llegan derecho al pan", "es algo de crianza" y "por comodidad".

Al consultar sobre qué mensajes podrían convencerlas de lo importante que es comer frutas y verduras, señalaron "el peso, todas estamos siempre pensando en que tenemos que bajar de peso", y respecto a las características de esos mensajes indicaron "tiene que ser algo que te impacte", "que sea llamativo".

Respecto a las etapas del cambio para la realización de actividad física, en la tabla 3 se observa que el 4,3\% y el $18,6 \%$ de las madres se encontraban en las etapas de pre-contemplación y contemplación, respectivamente. El 25,1\% indicó encontrarse en la etapa de preparación para la acción, el 18,9\% en acción y el 33,1\% en mantenimiento.
Las motivaciones y barreras respecto a la realización de actividad física también fueron analizadas según estado nutricional, por las razones ya señaladas. En la tabla 4 destacan como motivaciones: "me hace sentir bien", "para mantener mi salud", "para liberar tensiones" y "para perder peso", sin diferencias significativas según estado nutricional. "para mantenerme en forma" y "para mantener mi peso actual" mostraron una significativa mayor proporción de respuestas en las mujeres de peso normal respecto a las con sobrepeso y obesidad $(\mathrm{p}<0,05)$.

En relación a las barreras, la respuesta más frecuente fue "no tengo tiempo", seguida de lejos por "no estoy acostumbrada" y "sale muy caro ir a un gimnasio", sin diferencias significativas según estado nutricional. Una mayor proporción de madres con sobrepeso y obesidad señaló "no tengo quien me acompañe" $(\mathrm{p}<0,05)$.

En los grupos focales sobre percepciones, creencias y valores relacionados con la actividad física, al consultarles si harían ejercicio en su tiempo libre, aunque señalaron "sí, hay que hacerlo, es tan necesario como una alimentación sana", los planteamientos más frecuentes se refirieron a las dificultades: "sí, pero cuesta" y "uno es floja en eso". Como actitud positiva destacó "estoy tratando de caminar más y subir las escaleras del metro, no las mecánicas”. Ante la consulta ¿Qué las motivaría a hacer ejercicio? Las respuestas fueron "bajar de peso", "ver resultados, así me darían ganas de seguir". Entre las actividades que prefieren realizar en lugar de ejercicios para sentirse bien, los comentarios más frecuentes fueron "ver televisión", "comer algo rico", "juntarme a conversar con alguien"; o "estar en al computador, es un vicio".

\section{DISCUSIÓN}

Los resultados de este estudio muestran una prevalencia creciente de obesidad en los niños a medida que aumenta la obesidad de la madre. Sin embargo, la

\section{TABLA 3}

Etapas del cambio en actividad física en madres de preescolares. $(\mathrm{N}=320)$.

\section{Etapas del cambio}

Precontemplación

Contemplación

Preparación para la acción

Acción

Mantenimiento

Total

\section{$\mathbf{N}$}

14

58

80

61

107

320
$\%$

4,3

18,6

25,1

18,9

33,1

100,0 
elevada prevalencia de sobrepeso observada en todos los niños, independientemente del estado nutricional de sus madres, plantea la necesidad de estudiar los comportamientos e implementar intervenciones educativas que las involucren a todas. Esto, teniendo en cuenta que si bien se reconoce el papel fundamental de la familia, en especial de la madre, en la formación de hábitos en sus hijos (19-21), son escasos los estudios nacionales sobre sus actitudes y conductas respecto a la alimentación saludable y la actividad física $(28,34)$.

Sólo el 5\% de las madres cumplía con la recomendación actual de la OMS sobre el consumo de F\&V. La mayoría (61\%), comía 1 a 2 porciones diarias. Estos resultados son semejantes a los encontrados por Olivares y cols., en estudiantes universitarios y madres de escolares $(27,28)$, y significa que las estrategias utilizadas hasta la fecha, de promover el consumo de F\&V a través de las guías alimentarias (14), o las actividades realizadas por la Corporación "5 al Día Chile" $(11,24)$, no han logrado tener efecto en el grupo estudiado.

Al analizar las etapas del cambio en el grupo que consumía 1 a 2 porciones, el $95 \%$ manifestó encontrarse en la etapa de preparación para la acción, es decir, con la intención de aumentar su consumo en una fecha próxima. Sin embargo, tanto la Corporación 5 al Día Chile, como estrategias internacionales diseñadas para promover el consumo de 5 porciones, han encontrado como barrera común el que las personas que comen 1 a 2 porciones

\section{TABLA 4}

\section{Motivaciones y barreras de madres de preescolares para realizar al menos 30 minutos de actividad física 5 veces por semana, según estado nutricional.}

\begin{tabular}{|c|c|c|c|c|c|c|}
\hline \multirow{2}{*}{ MOTIVACIONES } & \multicolumn{2}{|c|}{$\begin{array}{c}\text { Peso normal } \\
n=116\end{array}$} & \multicolumn{2}{|c|}{$\begin{array}{c}\text { Sobrepeso y obesas } \\
\qquad n=204\end{array}$} & \multicolumn{2}{|c|}{$\begin{array}{c}\text { Total } \\
\mathbf{n}=\mathbf{3 2 0}\end{array}$} \\
\hline & $\mathbf{n}$ & $\%$ & $\mathbf{n}$ & $\%$ & $\mathbf{n}$ & $\%$ \\
\hline Me hace sentir bien & 62 & 53,5 & 107 & 52,5 & 169 & 52,8 \\
\hline Para mantener mi salud & 51 & 44,0 & 111 & 54,4 & 162 & 50,6 \\
\hline Me sirve para liberar tensiones & 53 & 45,7 & 100 & 49,0 & 153 & 47,8 \\
\hline Para perder peso & 28 & 24,1 & 117 & 57,4 & 145 & 45,3 \\
\hline Para mantenerme en forma & 61 & 52,6 & 82 & 40,2 & 143 & $44,7 *$ \\
\hline Me gusta & 43 & 37,1 & 66 & 32,5 & 109 & 34,1 \\
\hline Me entretiene & 40 & 34,5 & 50 & 55,6 & 90 & 28,1 \\
\hline Para mantener mi peso actual & 40 & 34,5 & 32 & 15,7 & 72 & $22,5^{*}$ \\
\hline Me permite compartir con amigos & 19 & 16,4 & 36 & 17,7 & 55 & 17,2 \\
\hline Tengo amigos que me acompañan & 14 & 12,1 & 21 & 10,3 & 35 & 10,9 \\
\hline \multicolumn{7}{|l|}{ BARRERAS } \\
\hline & $\mathbf{n}$ & $\%$ & $\mathbf{n}$ & $\%$ & $\mathbf{n}$ & $\%$ \\
\hline No tengo tiempo & 63 & 54,3 & 112 & 54,9 & 175 & 54,7 \\
\hline No estoy acostumbrada & 24 & 20,7 & 51 & 25,0 & 75 & 23,4 \\
\hline Sale muy caro ir a un gimnasio & 22 & 19,0 & 47 & 23,0 & 69 & 21,6 \\
\hline Llego muy cansada del trabajo & 20 & 17,2 & 47 & 23,0 & 67 & 20,9 \\
\hline No tengo quien me acompañe & 14 & 12,1 & 43 & 21,1 & 57 & $17,8^{*}$ \\
\hline Por flojera & 15 & 12,9 & 38 & 18,6 & 53 & 16,6 \\
\hline No tengo con quien dejar a mis hijos & 9 & 7,8 & 21 & 10,3 & 30 & 9,4 \\
\hline No hay seguridad en la calle & 6 & 5,2 & 16 & 7,8 & 22 & 6,9 \\
\hline Tengo problemas de salud & 6 & 5,2 & 16 & 7,8 & 22 & 6,9 \\
\hline No me gusta & 6 & 5,2 & 10 & 4,9 & 16 & 5,0 \\
\hline Prefiero la TV o el computador & 6 & 5,2 & 11 & 5,4 & 17 & 5,3 \\
\hline
\end{tabular}


consideran que ya comen lo suficiente $(11,12,24)$. El precio también fue considerado una barrera, lo que puede tener relación con el período en el que fueron encuestadas las madres, un invierno en el que se registraron alzas importantes en el valor de estos productos. Chile tiene una gran disponibilidad de frutas y verduras a precios accesibles, por lo que el precio habitualmente no es un factor limitante (35). Sin embargo, esta barrera es mencionada tanto a nivel nacional como internacional, en especial por las personas de NSE bajo (36).

En cuanto a la actividad física, el estudio muestra que el $52 \%$ de las madres se encuentran en las etapas de acción y mantenimiento (18,9\% y $33,1 \%$ respectivamente). Considerando la alta prevalencia de sobrepeso y obesidad en este grupo (63\%), esta situación podría interpretarse como una sobreestimación de la actividad por el grupo estudiado. En nuestro país, el año 2001 se promulgó la nueva Ley del Deporte (37) y el año 2003 el Ministerio de Salud publicó la Guía para una Vida Activa (38), con el fin de promover el deporte y la actividad física en el país. Sin embargo, la última Encuesta Nacional de Salud (3) muestra que las cifras de sedentarismo siguen siendo altas (4), lo que plantea la necesidad de nuevos enfoques para abordar el problema. No se han identificado intervenciones dirigidas a madres de preescolares en el país, las que en este estudio manifestaron diversas motivaciones importantes para ellas, semejantes a las encontrados por Rodríguez-Romo y cols., en mujeres de Madrid (39) y por Anderson en mujeres norteamericanas (40).

En los grupos focales se observó que tanto en actividad física como en alimentación saludable las madres demostraron tener conocimientos, asociando la alimentación saludable con el consumo de frutas y verduras, al igual que en los estudios de Eikenberry y cols. (29) y Borra y cols. (30). Es importante señalar que si estos conocimientos no se traducen en cambios de conducta, es necesario considerar sus motivaciones para lograr avances en ambos temas, y contar con el apoyo de políticas y programas que consideren esa disociación.

Las madres reportaron la necesidad de mayor información y explicaciones que las motiven a actuar. Explicitaron que una forma de captar la atención, tanto de ellas como de sus hijos, sería por medio de mensajes llamativos y que apunten a lo que a ellas les importa, como por ejemplo "bajar de peso" y no a consecuencias que ven muy lejanas como la prevención de enfermedades. En lo que respecta a mensajes para aumentar el consumo de verduras y frutas, esto coincide con lo planteado por Lefebvre y cols. (41), Kotler y cols. (15), el Centro para la Prevención y Control de Enfermedades de los Estados Unidos (16), Stead y cols. (42), y otros, quienes recomiendan realizar investigación formativa para el diseño de mensajes significativos para cada grupo y el desarrollo de estrategias de mercadeo social, que utilicen el modelo ecológico, como las usadas en Estados Unidos, Canadá, Australia y otros países desarrollados.

Desde el año 2006, la OPS/OMS está promoviendo que los países de América Latina utilicen estrategias de mercadeo social para promover el consumo de frutas y verduras, aumentar la actividad física y prevenir la obesidad (18).

Este estudio no incluyó la ingesta de alimentos, en especial la de aquellos que aumentan la densidad energética de la dieta, lo que puede ser considerado una limitante que debería ser superada en estudios posteriores. La actividad física puede haber sido sobreestimada por las madres, aunque es posible que por la edad de sus hijos, efectivamente tengan una mayor demanda de la misma, si debido a su NSE no cuentan con ayuda para su cuidado.

Este trabajo intenta contribuir al fomento de estudios de investigación formativa que sirvan de línea base a las estrategias de promoción de salud, educación o mercadeo social. Identificar las etapas del cambio de conducta en la que se encuentran las madres de los preescolares permitiría segmentar los grupos con mayor disposición al cambio con los que se recomienda trabajar. Sus motivaciones y barreras para aumentar el consumo de frutas y verduras y la actividad física, podrían ser útiles para el diseño de mensajes e intervenciones específicas para este importante grupo, ya que un cambio positivo en sus conductas actuales podría contribuir a mejorar la creación de hábitos saludables en sus hijos.

\section{RESUMEN}

Con el fin de diseñar intervenciones efectivas que contribuyan a prevenir la obesidad infantil, se determinaron las etapas del cambio de la conducta, las motivaciones y barreras para consumir 5 porciones diarias de frutas y verduras y realizar actividad física en 323 madres de preescolares atendidas en Centros de Atención Primaria de Salud, mediante la aplicación de una encuesta validada en estudios previos. Sólo el 5\% de ellas consumía 5 o más porciones diarias, la mayoría consumía 1 a 2 porciones $(61,3 \%)$. Las principales motivaciones para comer 5 porciones fueron: "me gustan" y "para perder peso". La principal barrera "se me olvida comerlas". En la actividad física, el 33,1\% indicó encontrarse en la etapa de mantenimiento y el 18,9\% en acción. Las principales motivaciones: "me hace sentir bien" y "para perder peso". La principal barrera: "no tengo tiempo". Se sugiere que las intervenciones educativas consideren las actitudes y creencias de las madres, para ser más 
efectivas.

Palabras clave: Madres de preescolares, motivaciones, barreras, consumo de frutas y verduras, actividad física.

Dirigir la correspondencia a:

Nutricionista

Lorena Meléndez I.

Apoquindo 7750 depto. 2203, Las Condes

Santiago

Chile

Fono: 56-92187999

e-mail: lmelendez@inta.uchile.cl

\section{BIBLIOGRAFÍA}

1. World Health Organization. Diet, nutrition and the prevention of chronic diseases: Report of a Joint WHO/FAO Expert Consultation. Geneva: WHO; 2003 (WHO Technical Report Series 916).

2. Organización Mundial de la Salud (OMS). Estrategia mundial sobre régimen alimentario, actividad física y salud. $57^{\text {a }}$ Asamblea Mundial de la Salud. WHA57.17. Ginebra: OMS; 2004.

3. Ministerio de Salud. Encuesta_ Nacionalde Sald 2009-2010 Disponible en http./www redsalud govel/portal/m/item/99c12b89738d80d5e04001 011e0113f8.pdf

4. Ministerio de Salud. III Encuesta Nacional de Salud. Santiago: MINSAL; 2003.

5. Gobierno de Chile. Ministerio de Salud. II Encuesta de Calidad de Vida y Salud 2006 Santiago. Minsal2007 Disponible en http./epi minsal cl/epi/html/ sdesalud/calidaddevida2006/index.htm

6. Ministerio de Educación. Junta Nacional de Auxilio Escolar y Becas. Estado nutricional de niños que ingresan a primer año hásico Disponible en URI http://zeus.junaeb.cl/MapaNutricionalGx/Acceso 1 de abril de 2011.

7. Ministerio de Salud. Situación nutricional en el sistema público de salud 2007-2009. Santiago: MINSAL; 2010.

8. Junta Nacional de Jardines Infantiles (JUW) Centro de documentación. Disponible en: Www.junji. c1. Serie de Documentos Técnicos.

9. Kain J, Lera L, Rojas J, Uauy R. Obesidad en preescolares de la Región Metropolitana de Chile. Rev Med Chile 2007; 135: 63-70.

10. World Cancer Research Fund/American Institute for Cancer Research. Food, nutrition and the prevention of cancer: a global perspective. Washington DC: WCRF/AICR; 1997.

11. Vio F, Zacarías I, González D. Implementación de un programa de promoción del consumo de frutas y verduras. Corporación 5 al Día Chile. En: S Olivares, M Leporati, Villalobos P, L Barría, eds. Contribución de la política agraria al consumo de frutas y verduras en Chile: un compromiso con la nutrición y la salud de la población. Santiago: Ministerio de Agricultura/INTA/Corporación 5 al Día Chile; 2008.

12. Zacarías I, Keller I, editoras. Programa Internacional 5 al Día y su aplicación en diferentes países de América Latina. Rev Chil Nutr 2006; 33(Supl 1): 224-319.

13. Salinas J, Vio F. Política de promoción de salud en Chile. En: C Albala, S Olivares, J Salinas, F Vio, eds. Bases, prioridades y desafíos de la promoción de la salud. Santiago: Ministerio de Salud/ Vida Chile/Universidad de Chile/INTA, Andros Impresores; 2004.

14. Ministerio de Salud/INTA/Vida Chile. Guía para una Vida Saludable. Santiago: Ministerio de Salud; 2005. Norma 76. Disponible en: http://www.inta.cl/ materiales educativos.

15. Kotler Ph, Lee N. Social marketing. Influencing behaviors for good. 3rd edition. California: Sage Publications; 2008.

16. Center for Disease Control and Prevention. National Center for Chronic Disease Prevention and Health Promotion Atlanta. CDC; 2007 Disponible en http://www.cdc.gov.youthcampaign

17. Prochaska J, Velicer W, Rossi J, Goldstein M, Marcus B et al. Stages of change and decisional balance for twelve problem behaviours. Health Psychol 1994; 13:39-46.

18. PAHO/WHO. Regional communication plan 20062008. Let's eat healthy, live well, and get moving America! Washington DC: PAHO; 2006.

19. Boutelle K, Birkeland R, Hannan P. Associations between maternal concern for healthful eating and maternal eating behaviors, home food availability, and adolescent eating behaviors. J Nutr Educ Behav 2007; 39: 248-56.

20. Domínguez P, Olivares S, Santos JL. Influencia familiar sobre la conducta alimentaria y su relación con la obesidad infantil. Arch Latinoam Nutr 2008; 58(3): 249-55.

21. Chang M, Nitzke S, Guilford E, Adair C, Hazard D. Motivators and barriers to healthful eating and physical activity among low-income overweight and obese mothers. J Am Diet Assoc 2008; 108:1023-8.

22. Adimark GfK. Investigación de mercados y opinión pública. El nivel socioeconómico Esomar. Manual de aplicación. Santiago: Adimark GfK; 2000. 
23. National Institutes of Health/National Cancer Institute. 5 a Day for Better Health Program. Washington DC: Government Printing Office-Monograph; 2004.

24. Zacarías I, Pizarro T, Rodríguez L, González D, Domper A. Programa 5 al día para promover el consumo de frutas y verduras en Chile. Rev Chil Nutr 2006; 33(2): 276-80.

25. Marcus B, Selby V, Niaura R, Rossi J. Self-efficacy and the stages of exercise behavior change. Res Q Exerc Sport. 1992; 63: 60-6.

26. Escobar MC, Olivares S, Zacarías I. Manejo alimentario del adulto con sobrepeso y obesidad. Santiago: Ministerio de Salud; 2002.

27. Olivares S, Lera L, Bustos N. Etapas del cambio, beneficios y barreras en actividad física y consumo de frutas y verduras en estudiantes universitarios de Santiago de Chile. Rev Chil Nutr 2008; 35(1): 25-35.

28. Olivares S, Lera L, Mardones MA, Araneda J, Olivares MA, Colque ME. Motivaciones y barreras para consumir 5 porciones diarias de frutas y verduras al día en madres de escolares y profesores de enseñanza básica. Arch Latinoam Nutr 2009; $59(2) \cdot 166-73$

29. Eikenberry N Smith C. I Am Diet Assoc. Healthful eating: perceptions, motivations, barriers, and promoters in low-income Minnesota communities.

30. Borrast, Kelly L, Shirreffs MB, Neville K, Geiger CJ. Developing health messages: qualitative studies with children, parents, and teachers help identify communications opportunities for healthful lifestyles and the prevention of obesity. I Am Diet Assoc 2003; 103(6):721-8.

31. World Health Organization. Obesity. Preventing and managing the global epidemic. Report of a WHO Consultation on Obesity. Geneva: WHO; 1997.

32. Organización Mundial de la Salud. Patrones de crecimiento infantil de la OMS. Ginebra, 2006.
Disponible en: http://www.who.int/childgrowth/en/

33. STATA. Stata 10.1. Stata Corporation. USA: College Station; 2008.

34. Olivares S, Bustos N, Moreno X, Lera L, Cortez S. Actitudes y prácticas sobre alimentación y actividad física en niños obesos y sus madres en Santiago, Chile. Rev Chil Nutr 2006; 33(2): 170-9.

35. Ministerio de Agricultura. Oficina de Estudios y Políticas Agrarias (ODEPA). Exportación de frutas y hortalizas. Santiago: ODEPA; 2007. Disponible en http://www.odepa.gob.cl

36. Cassady D, Jetter K, Culp J. Is Price a barrier to eating more fruits and vegetables for low-income families? J Am Diet Assoc 2007; 107: 1909- 15.

37. Gobierno de Chile. Política nacionalde_actividad fúsica y deporte 2001 http://www.chiledeportes. gov.cl/pdfs/lapolitica.pdf

38. Gobierno de Chile. Ministerio de Salud, Ministerio de Educación, Vida Chile, INTA, Universidad de Chile, Chile Deportes. Guía para una vida activa. Santiago; Andros Impresores: 2003.

39. Rodríguez-Romo G, Boned-Pascual C, GarridoMuñoz M. Motivos y barreras para hacer ejercicio y practicar deportes en Madrid. Rev Panam Salud Pública 2009; 26(3): 244-54.

40. Anderson C. When more is better: number of motives and reasons for quitting as correlates of physical activity in women. Health Educ Res 2003; 18(5): 525-37.

41. Lefebvre C, Doner L, Johnston C, Loughrey K, Balch G, Sutton Sh. Use of database marketing and consumer-based health communication in message design. In: Maibach E, Parrott R Eds. Designing health messages. Approaches from communication theory and public health practice. London: Sage Publications; 1995.

42. Stead M, Gordon R, Angus K. A systematic review of social marketing effectiveness. Health Education 2007; 107 (2): 126-91. 